\title{
Defectos en las Instalaciones Domésticas con Agua Debido a Disputas Legales: El Caso de Barcelona
}

\author{
M.J. Carretero-Ayuso $^{1 *}$, M $^{\text {a }}$.T. Pinheiro-Alves ${ }^{2}$, J.D. Bienvenido-Huertas ${ }^{3}$ y D. Marín-García ${ }^{4}$ \\ *Autor de Contacto: carreteroayuso@yahoo.es \\ ${ }^{1}$ Fundación-Musaat y Escuela de Arquitectura, Universidad de Alcalá, Alcalá de Henares, España \\ ${ }^{2}$ Dpto. de Arquitectura, Escuela de Artes y Arquitectura, Universidad de Évora, Évora, Portugal \\ ${ }^{3}$ Dpto. de Construcciones Arquitectónicas II, ETS Ingeniería Edificación, Universidad de Sevilla, Sevilla, España \\ ${ }^{4}$ Dpto. de Expresión Gráfica e Ing. Edificación, ETS Ingeniería Edificación, Universidad de Sevilla, Sevilla, España
}

\section{RESUMEN}

Esta investigación permite conocer los defectos existentes en instalaciones con canalizaciones de agua de tres variantes de tipología constructiva residencial. Se recurre a informes periciales edificatorios debidos a las demandas de usuarios ante los tribunales. Se determinaron cuáles son las anomalías y los orígenes del fallo que actúan como agentes causales. Para cada uno de ellos se determinaron los casos presentes en la instalación en donde se ubican, así como las situaciones más recurrentes. Se desglosaron uno por uno todos los procesos patológicos que se constataron, los cuáles se definen como la interrelación constructiva entre los parámetros estudiados. De esta forma, los diferentes intervinientes en el sector edificatorio dispondrán del know-how necesario para evitar a futuro los errores de obra.

Palabras clave: daños, causas, anomalías, procesos patológicos. 


\title{
Defects in Domestic Water Facilities Due to Legal Disputes: The Case of Barcelona
}

\author{
M.J. Carretero-Ayuso, Ma .T. Pinheiro-Alves, J.D. Bienvenido-Huertas y D. Marín-García
}

\begin{abstract}
The objective of this research is to know the defects that occur in water facilities in three types of residential construction typologies. Expert reports attached to court complaints filed by building users were reviewed. The types of anomalies that occur were determined, as well as the origins of the errors acting as causal agents of said defects. For each one, the number of cases occurring in each facility was determined and an analysis of the most recurring situations was carried out. Lastly, each of the observed pathology processes was broken down - processes defined as the construction interrelation between the studied parameters. Furthermore, the different participants in the construction sector will possess the necessary know-how to prevent future construction errors.
\end{abstract}

Key words: damages, causes, anomalies, error origin.

\section{Defeitos em Instalações Domésticas com Agua Devidos a Disputas Legais: O Caso de Barcelona}

\author{
M.J. Carretero-Ayuso, Ma.T. Pinheiro-Alves, J.D. Bienvenido-Huertas y D. Marín-García
}

\begin{abstract}
RESUMO
O objectivo desta investigação é o de identificar os defeitos que surgem nas instalações com água em três variantes de tipologia construtiva residencial. Recorre-se aos relatórios periciais associados aos registos das queixas de tribunal feitas pelos utilizadores dos edifícios. Foram determinados os tipos de anomalias que ocorrem, bem como as origens dos defeitos que actuaram como agentes causais dos mesmos. Para cada um, determinou-se os casos em cada instalação e foi feita a análise de quais são as situações mais recorrentes. Foram discriminados todos os processos patológicos que foram constatados, os quais se definem como a interrelação construtiva entre os parâmetros estudados. Aliás, os diferentes intervenientes do sector construtivo disporão do know-how necessário para evitar errores de obra no futuro.
\end{abstract}

Palavras chave: danos, causas, anomalias, origem do defeito. 


\section{INTRODUCCIÓN}

Los vicios ocultos en los edificios constituye uno de los temas que ha provocado más desasosiego a los ciudadanos y el que en mayor medida ha revelado la diferencia de fuerzas entre el promotorvendedor y el usuario-adquirente (Gallego Brizuela, 2011). Las presiones y prisas para entregar cuanto antes un edificio y los problemas de coordinación con los subcontratistas, puede dar lugar a multitud de anomalías y defectos. Por esta razón, el énfasis en la supervisión y en el control de calidad de las operaciones de subcontratación de las unidades de obra es fundamental para asegurar que estas problemáticas se vean reducidas (Forcada et al., 2013).

Tras realizar una encuesta sobre 74 edificios, Chong and Low (2006) indicaron que las anomalías suelen repetirse, lo que hace muy necesario que se apliquen en los estudios de arquitectura aquellas técnicas que hagan implementar la adquisición de conocimientos a través de la retroalimentación (lesson-learned) para mejorar la calidad final de los proyectos. Forcada et al. (2013) realizaron un análisis de multitud de viviendas en España, constatando que determinados tipos de anomalías no se suelen detectar y resolver hasta que llega la hora de la primera ocupación, lo que hace que sus reparaciones sean más costosas.

Una gran parte de las investigaciones consultadas se han centrado en procesos de observación y encuestas, no en casos reales. Al diagnosticar una anomalía existente es común encontrar que las causas primitivas que originaron los casos se siguen repitiendo en los actuales procesos de trabajo, por lo que es adecuado reconocerlas para mejorar estas unidades constructivas (Meiss and Muñoz, 2015). En base a lo indicado por Josephson and Hammarlund (1999) podrían disminuirse los costos asociados por falta de calidad, concentrando la atención sobre ellos durante la fase de puesta en obra. Como se ve, a pesar de los años transcurridos por ciertas investigaciones, sus conclusiones siguen siendo válidas más de 20 años después.

Min et al. (2016) determinaron que las operaciones proactivas y las estrategias de mantenimiento en los edificios existentes pueden contribuir a mejorar significativamente el rendimiento de éstos y que los procesos de diseño integrados deben ampliarse para incluir el control operativo de facility manager y la gestión de una manera global.

Según el Laboratorio de Edificación de la Escuela Politécnica de Edificación de Barcelona, el 5\% del coste operativo de un edificio corresponde al proyecto, el $20 \%$ a la construcción, el $65 \%$ al mantenimiento y gastos de explotación, y el 10\% coste de la rehabilitación o derribo (García SanzCalcedo, 2014). Por otro lado, el coste de las instalaciones en un edificio residencial, supone entre un 25 y un 35\% del valor de la construcción del mismo y ocupan el 7,7\% del espacio útil del mismo (Martín-Gómez et al., 2006).

Aunque una parte de los problemas habituales en la edificación provienen de un incorrecto diseño de los edificios y de sus instalaciones, la falta o la inadecuada revisión periódica de las instalaciones es también un problema que afecta a una gran parte del parque inmobiliario (Arencibia, 2007). Esto hace que el mantenimiento se convierta en un parámetro clave que permita que se llegue o se supere adecuadamente el periodo de vida útil del edificio en condiciones óptimas de funcionamiento (AENOR, 2012), y es la herramienta imprescindible para garantizar las condiciones de diseño y de correcta gestión (Min et al., 2016).

Antes de la crisis económica y constructiva que ha venido padeciendo España desde 2008, el sector de la construcción podría representar el 18\% del producto interior bruto (Azorín López and Monjo Carrió, 2005) lo que la convertía en el primer sector industrial del país. Teniendo en cuenta además, que la compra de una vivienda es la mayor inversión que un ciudadano medio hace durante toda su vida, se entiende el pensamiento de algunos autores (Blanchére, 1978) de que una de las exigencias reiteradas de las personas respecto a las construcciones es que no cuesten más que un precio determinado y que su mantenimiento sea asimismo de costo limitado. Por esta razón, y con el objeto de no judicializar en exceso las situaciones donde el importante precio pagado no exime de 
padecer defectos en los inmuebles adquiridos, se han iniciado en algunas ciudades de España un servicio de mediación técnico-profesional donde los arquitectos técnicos se ofrecen como intermediaros expertos para solucionar de manera amistosa las disconformidades entre clientes y promotoras o constructoras (Fernández 2015). Estas iniciativas pueden llegar a ser interesantes dado que según la Organización de Consumidores y Usuarios (OCU) la vivienda ha sido tradicionalmente el tercer sector español con más reclamaciones por parte de los ciudadanos (OCU, 2018), con unas 10.0000 quejas anuales.

Coincidimos con otros estudios (Garcez et al. 2012b) en la importancia de que los diseñadores sean especialmente meticulosos en la redacción de la documentación de los proyectos y que es necesario la contratación de mano de obra cualificada en la construcción de los edificios: solo estos dos aspectos, mejorarían ostensiblemente la calidad final de las edificaciones. Sobre este último aspecto, es reseñable que aun habiendo normativa española que indica los métodos y formas de puesta en obra de las instalaciones, en muchos casos éstas no se cumplen aun cuando los operarios pertenezcan a empresas calificadas como 'instaladores autorizados'.

Los edificios residenciales en España suponen un total de 3.500 millones de metros cuadrados, representando el $85 \%$ de la superficie construida (WWF-Spain, 2010). Todos los edificios tienen dotaciones de instalaciones, por lo que es importante que las mismas sean lo más fiables y eficaces posibles.

Sería conveniente que se implementaran de forma inminente, las nuevas tecnologías existentes (Pauwels 2014) en el proceso constructivo español, empezando por el diseño de los propios edificios, siguiendo por la contratación y la propia ejecución de las obras, así como durante el periodo de uso y mantenimiento de éstos (Lee et al., 2016). Una oportunidad para mejorar la calidad de todo el proceso edificatorio es la utilización de software BIM (Ministerio de Fomento, 2015), dado que sus características de transversalidad permitirían la reducción de costes y la mejora de los tiempos de producción (Chou et al., 2009).

\section{METODOLOGÍA}

Este estudio está fundamentado tomando como base documental los expedientes de responsabilidad civil de los arquitectos técnicos de España (MUSAAT, 2018). Todos estos datos proceden de reclamaciones judiciales interpuestas entre los años 2013 a 2017, con sentencia firme dictada en los años posteriores (SERJUTECA, 2013-2017). Es cuando la sentencia no es apelable, cuando los datos se introducen como casos para el estudio. Es por esta razón que hubieron de pasar varios años desde que se efectuó la demanda inicial para poder considerar cada uno de ellos en la base de datos.

En la investigación que aquí se presenta, el número de expedientes analizados relativos a las demandas efectuadas ante los tribunales fueron 242, que son aquellos correspondientes a los edificios residenciales de la provincia de Barcelona que reunían las condiciones antes mencionadas. En concreto, este artículo se centrará sobre las anomalías identificadas en los mismos relacionadas con las instalaciones de protección contra incendios, calefacción, fontanería y saneamiento.

Hay que tener en cuenta que los resultados aquí presentados tienen una aportación especial al campo del conocimiento científico: corresponden al 100\% del periodo de tiempo antes indicado, por lo que no se trata de una muestra más o menos parcial (no hay -por lo tanto- posibilidad de errores o desviaciones).

Dentro de las instalaciones se considerarán dos grandes grupos: las 'Instalaciones Con Canalizaciones de Agua' (ICCA), formadas por las cuatro instalaciones indicadas anteriormente, y las 'Instalaciones Sin Canalizaciones de Agua' (ISCA: electricidad, telecomunicaciones, ventilación, etc.). Según la determinación de los 242 casos constatados en la provincia de 
Barcelona, puede indicarse que el primer grupo concentra prácticamente las 2/3 partes del total $(\mathrm{ICCA}=160$ casos), mientras que el segundo está por encima levemente de una $1 / 3$ parte (ISCA= 82 casos).

En algunas de las investigaciones consultadas, se han estudiado cuáles son las anomalías que más se dan en una unidad de obra en concreto, respecto a un conjunto muy determinado de edificios (normalmente, relacionados con algún promotor o constructor específico). Esto hace que la extensión de las mismas sea parcial, abarcando solo algunas zonas de obra (Sarman et al., 2015); por esta razón el enfoque de este estudio es diferente (es toda una provincia) y su fuente de datos no tiene precedentes conocidos. Además de ello, se han incluido y analizado también el origen causal que provocaban esas anomalías; aspecto que no suele ser habitual en otros estudios, dado el esfuerzo que supone para los autores poder determinar dicho concepto.

Las instalaciones ICCA que han sido objeto de análisis y cuantificación en este estudio, son las que se indican en la Tabla 1.

Tabla 1 - Nombre de las instalaciones estudiadas y sus códigos

\begin{tabular}{|l|c|}
\hline Nombre de la instalación con canalizaciones de agua & Código \\
\hline Saneamiento & $\mathrm{I} 1$ \\
\hline Fontanería & $\mathrm{I} 2$ \\
\hline Calefacción & $\mathrm{I} 3$ \\
\hline Protección contra incendios & $\mathrm{I} 4$ \\
\hline
\end{tabular}

Sobre las citadas ICCA, se han determinado los diferentes tipos de anomalías que han sido objeto de reclamación judicial (Tabla 2).

Tabla 2 - Nombre de los tipos de anomalías estudiadas en ICCA y sus códigos

\begin{tabular}{|l|c|}
\hline Tipos de anomalías & Código \\
\hline Fugas en las conducciones & A1 \\
\hline Mal funcionamiento y/o atasco de la instalación & A2 \\
\hline Ausencia o deficiente disposición de piezas & A3 \\
\hline Desajustes y disfunciones & A4 \\
\hline Salida de malos olores & A5 \\
\hline
\end{tabular}

Del mismo modo, para cada uno de estos tipos de anomalías, se han explicitado cuáles son los tipos de 'origen del fallo' que dan lugar a las mismas. Se ha apreciado que existía una gran similitud entre ellos, independientemente de la instalación donde se ubicaran. Su tipificación queda descrita en la Tabla 3.

Tabla 3 - Nombre de los tipos de orígenes del fallo y sus códigos

\begin{tabular}{|l|c|}
\hline Tipo de origen del fallo & Código \\
\hline Daño en la instalación & OF1 \\
\hline Uniones/acoples deteriorados o mal colocados & OF2 \\
\hline Falta de colocación y/o omisión de elementos & OF3 \\
\hline Insuficiente porcentaje de pendiente & OF4 \\
\hline Inadecuado material o solución constructiva & OF5 \\
\hline Mantenimiento disconforme & OF6 \\
\hline
\end{tabular}


Del mismo modo, se adscribió - para cada uno de los casos estudiados- la tipología constructiva donde se producían las anomalías, la cual queda descrita en la Tabla 4.

Tabla 4 - Tipologías constructivas analizadas

\begin{tabular}{|l|c|}
\hline Nombre de la tipología constructiva & Código \\
\hline Edificación en altura & TO-1 \\
\hline Unifamiliar pareada/adosada & TO-2 \\
\hline Unifamiliar aislada & TO-3 \\
\hline
\end{tabular}

\section{RESULTADOS}

\subsection{Distribución de los casos por cada instalación.}

Una vez analizados los casos, la distribución de anomalías por cada instalación se indica en la Figura 1.

\section{I1 $=\mathrm{I} 2=\mathrm{I3}=\mathrm{I} 4$}

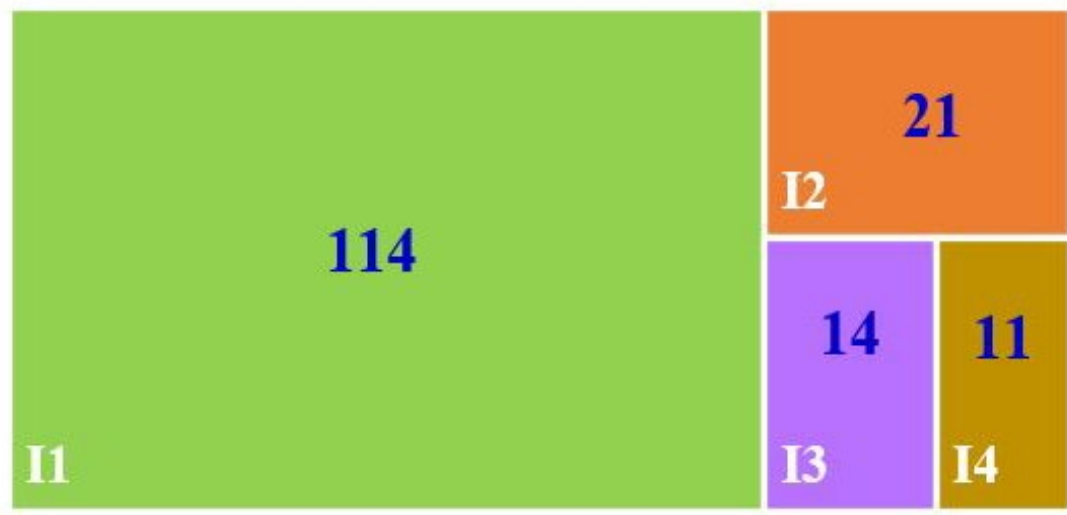

\section{Leyenda}

I1 - Saneamiento

I2 - Fontanería

I3 - Calefacción

I4 - Protección contra incendios

Figura 1. Distribución del número de casos por cada una de las instalaciones.

Como se aprecia, en 'Saneamiento' (I1) se concentra el 71\% del total, lo que hace que sea muy preocupante tanta concentración de anomalías en una sola instalación. Después de ella, le sigue la 'Fontanería' (I2) con el 13\% (21 casos).

\subsection{Distribución de casos en función de la tipología constructiva.}

Cuantificados los casos de anomalías por cada una de las tipologías constructivas donde se producían (Figura 2), se constató que la mayor repetición existía en las 'edificaciones en altura' (TO-1=62,74\%), seguido de las viviendas 'unifamiliares pareadas/adosadas' (TO-2=21,44\%). 


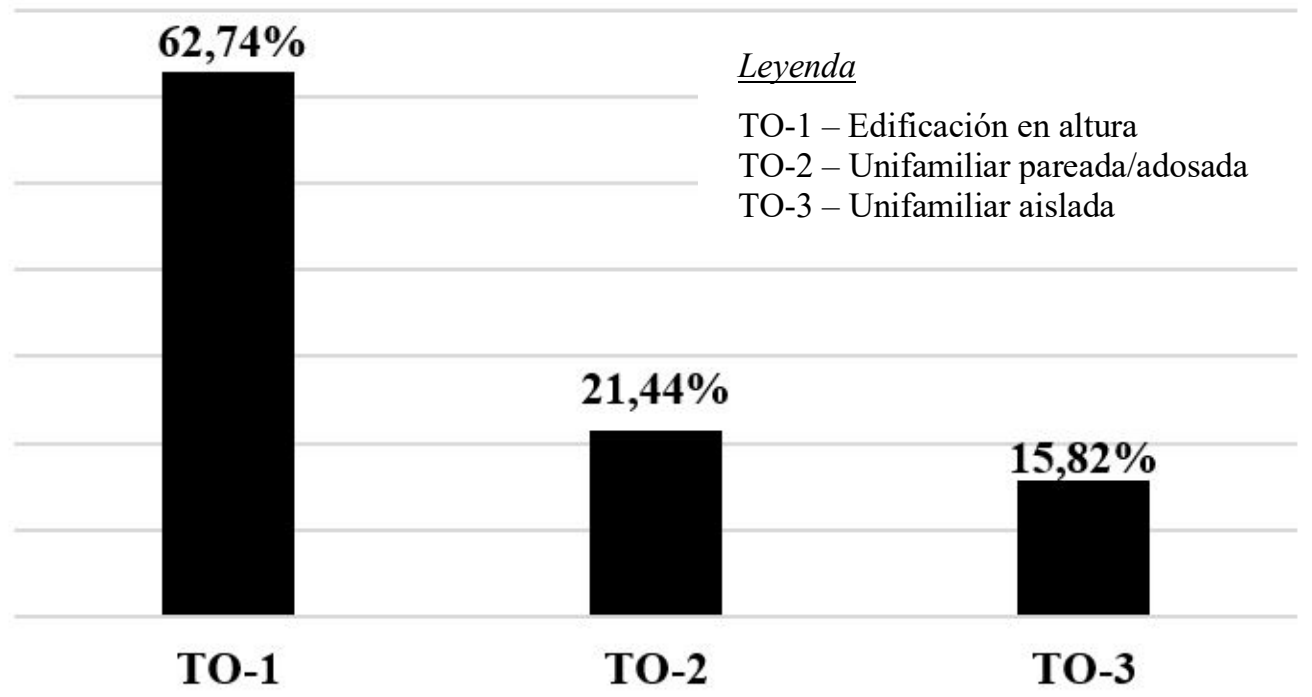

Figura 2. Distribución de casos según la tipología constructiva.

\title{
3.3 Distribución de casos según el origen del fallo.
}

Según se indicó en el capítulo de metodología, se tipificaron seis diferentes orígenes del fallo que daban lugar a las anomalías. En base a los resultados expresados en la Figura 3, se aprecia que los mayores valores se obtienen en 'daño en la instalación' (OF1=58 casos), 'uniones/acoples deteriorados o mal colocados ( $\mathrm{OF} 2=39$ casos) y 'falta de colocación y/o omisión de elementos' $(\mathrm{OF} 3=35$ casos $)$.
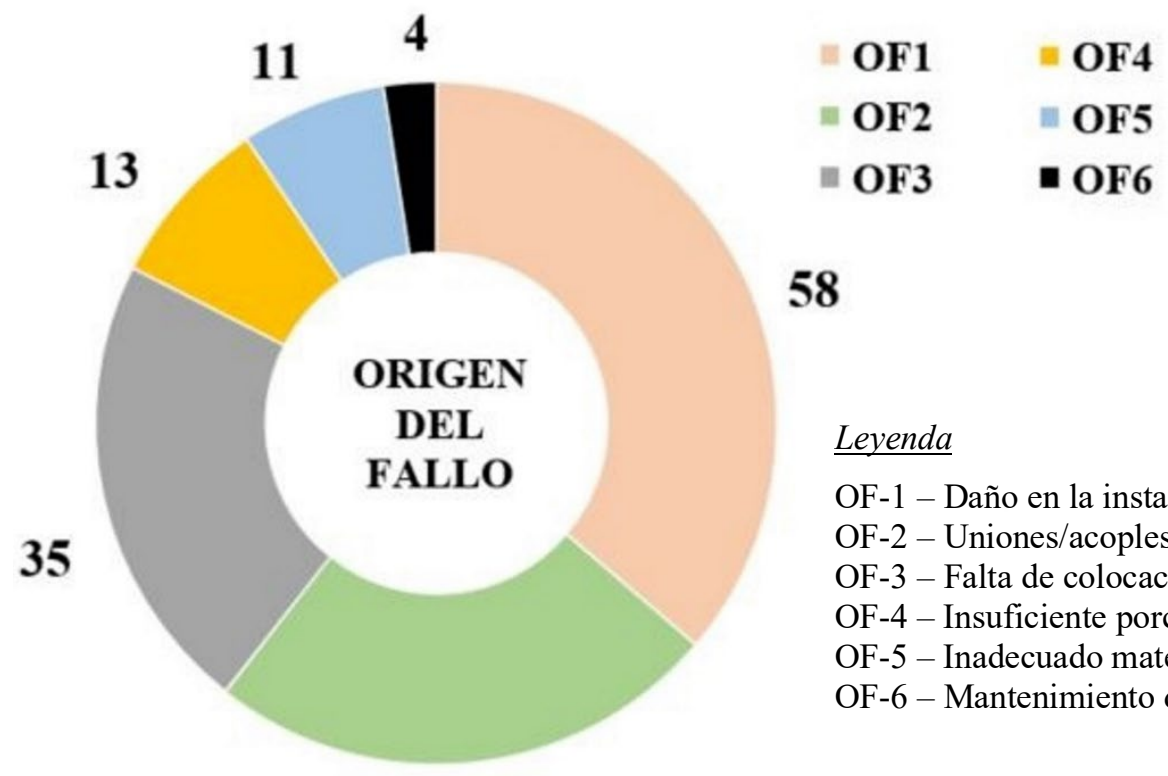

\section{8}

\author{
Leyenda \\ OF-1 - Daño en la instalación \\ OF-2 - Uniones/acoples deteriorados o mal colocados \\ OF-3 - Falta de colocación y/o omisión de elementos \\ OF-4 - Insuficiente porcentaje de pendiente \\ OF-5 - Inadecuado material o solución constructiva \\ OF-6 - Mantenimiento disconforme
}

\section{9}

Figura 3. Distribución de los casos según el origen del fallo. 


\subsection{Distribución de casos en función de los tipos de anomalías.}

Los cinco tipos de anomalías determinadas tienen una presencia desigual (ver Figura 4). De ellas, la que está presente en un mayor número de veces es 'fugas en las conducciones' (A1=72 casos), seguida de 'mal funcionamiento y/o atasco de la instalación' (A2=47 casos).

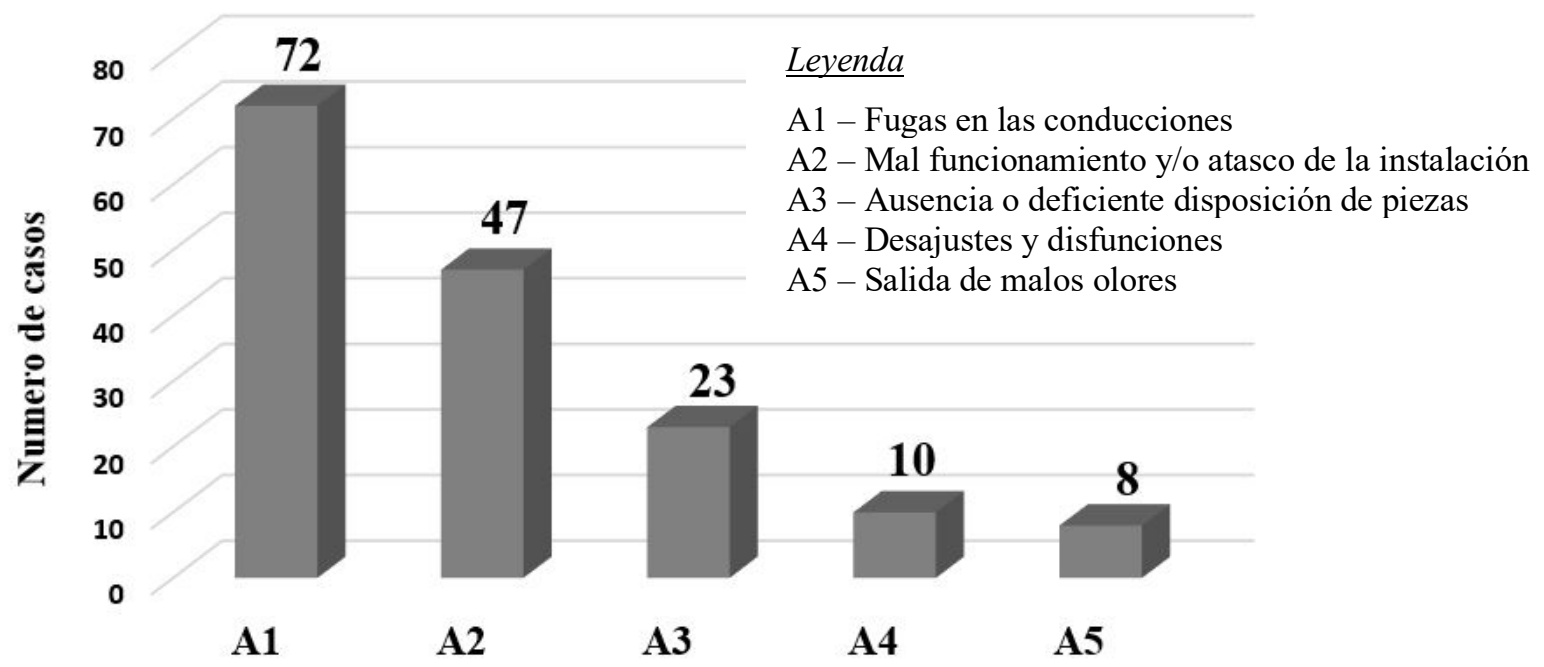

Figura 4. Distribución de casos existentes según el tipo de anomalía.

\subsection{Distribución según el conjunto de instalaciones.}

Haciendo un análisis detallado y descompuesto por cada uno de los tipos de anomalías, en base a los conjuntos de instalaciones ICCA e ISCA, se obtienen los valores de la Figura 5. Las anomalías en las que el peso de ICCA es totalmente dominante son A1 y A2.

Esto es, como se aprecia de la visión comparada de la Figura 4 y de la Figura 5, la distribución porcentual en el conjunto de las instalaciones (ICCA+ISCA) viene determinada por la preponderancia de anomalías existentes en ICCA por cada una de ellas. Es decir, cuanto mayor porcentaje de ICCA existe dentro de un tipo de anomalía dado, mayor preponderancia total tiene esa anomalía en el global estudiado.

\section{$\because$ ICCA $=$ ISCA}

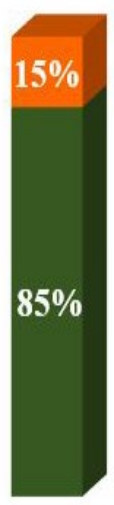

A1

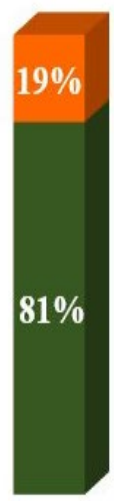

A2

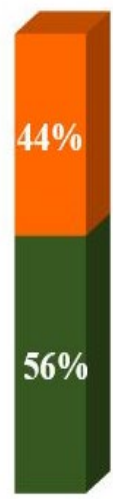

A3

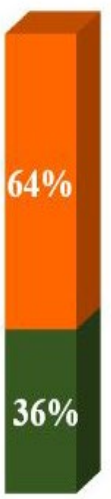

A4

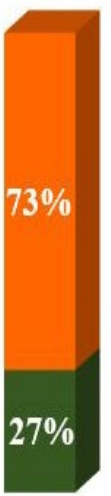

Leyenda

A1 - Fugas en las conducciones

A2 - Mal funcionamiento y/o atasco de la instalación

A3 - Ausencia o deficiente disposición de piezas

A4 - Desajustes y disfunciones

A5 - Salida de malos olores

ICCA - Instalaciones Con Canalizaciones de Agua

ISCA - Instalaciones Sin Canalizaciones de Agua

Figura 5. Distribución de casos según el tipo de anomalía y los conjuntos ICCA e ISCA. 
3.6 Número de casos por tipo de anomalías en fontanería, calefacción y contra incendios.

Se ha realizado un gráfico compuesto, desglosando el tipo y número de casos por tipo de anomalía y por tipo de instalación (Figura 6). En la parte superior de la Figura 6 se muestran los valores en fontanería, en la parte central de la Figura 6 están presentes los valores de la calefacción y en la parte inferior de dicha figura los valores de protección contra incendios.
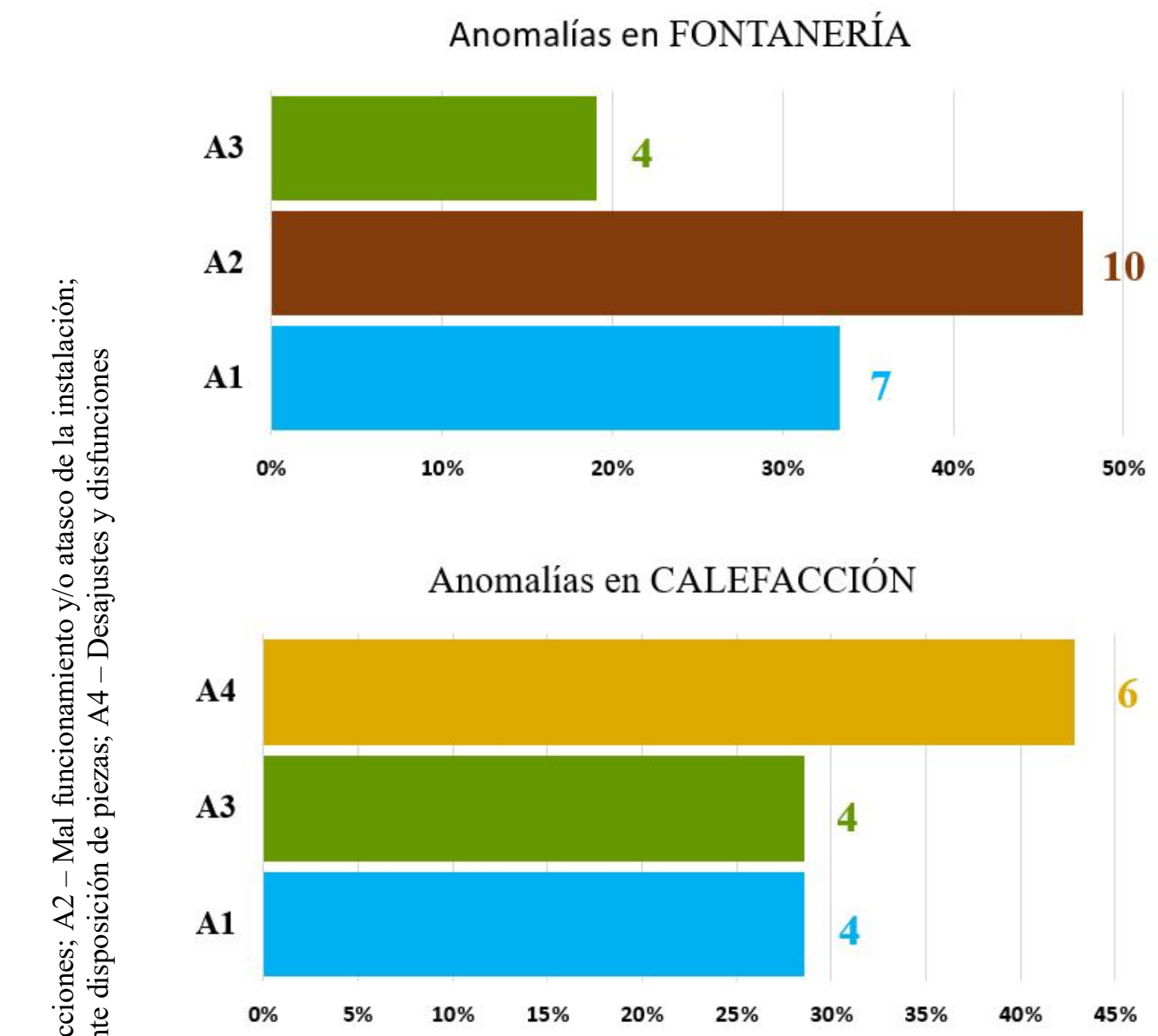

\section{Anomalías en PROTECCIÓN CONTRA INCENDIOS}

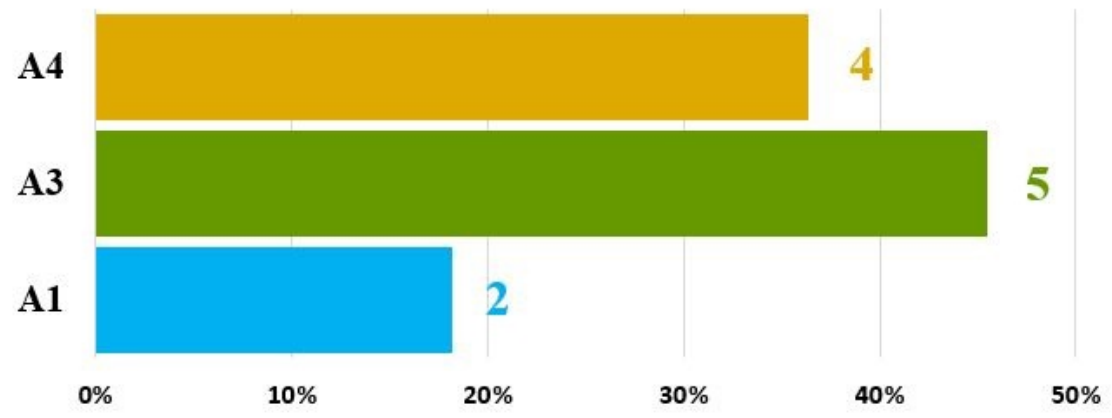

Figura 6. Número de casos por tipo de anomalía en fontanería, calefacción y protección contra incendios. 
Como se aprecia, A4 no está presente en la fontanería, mientras que A2 no está presente en la calefacción y en protección contra incendios. En ninguna de estas tres instalaciones se encuentra tampoco A5, la cual solo existe en el saneamiento.

\subsection{Presencia de casos por tipo de anomalías en el saneamiento.}

En concordancia con lo expresado en el apartado anterior, en la Figura 7 se recogen los datos para la instalación de saneamiento, en la cual la anomalía A1 es la más recurrente (59 casos), y en donde no existe A4 ('desajustes y disfunciones').

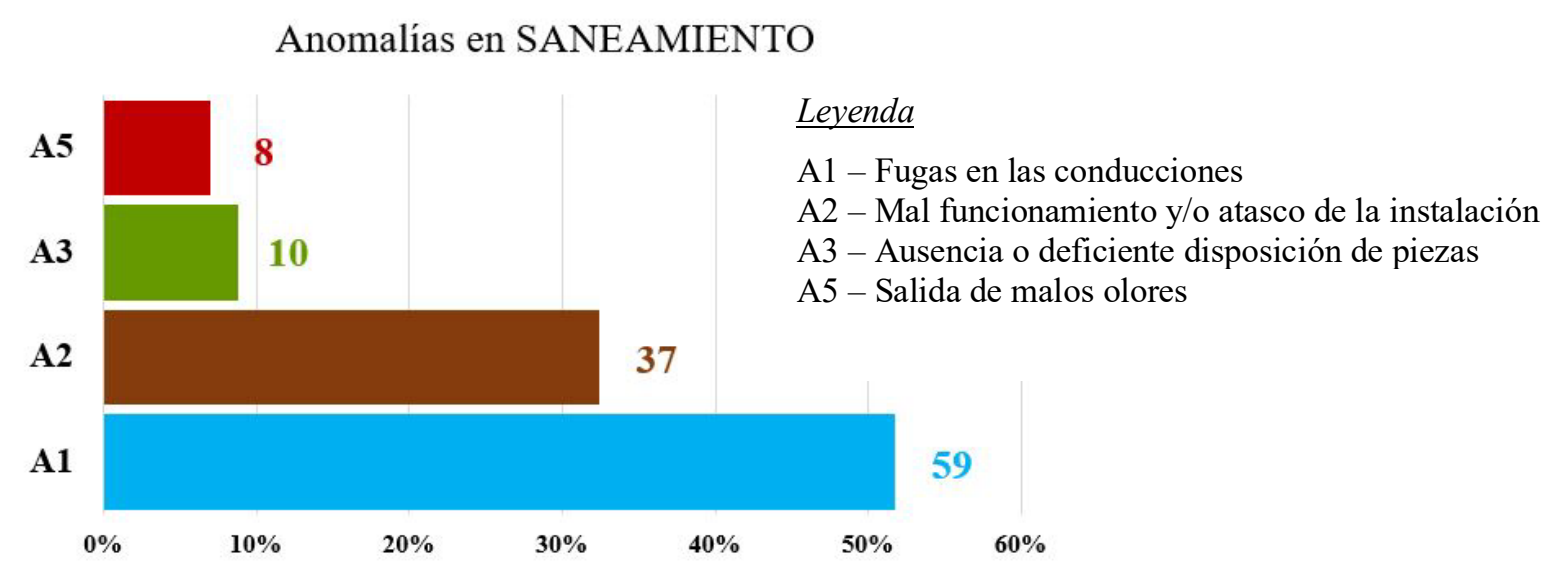

Figura 7. Número de casos por tipo de anomalía en la instalación de saneamiento.

Se han desgranado también los casos en función del subtipo de instalación de saneamiento; es decir, según se trate de 'Saneamiento Drenaje' (S.DRE), 'Saneamiento Colgado' (S.COL) y 'Saneamiento Enterrado' (S.ENT).

En la Figura 8 se incluyen los valores, tomando de base los subtipos de saneamiento y dentro de ellos los tipos de anomalías, mientras que en la Figura 9 se recogen estos mismos datos expresados a la inversa (por cada tipo de anomalía, su desglose por subtipo de instalación). Es significativo comprobar que el 'saneamiento drenaje' solo está presente en el tipo de anomalía A2 (mal funcionamiento y/o atasco de la instalación). Por su parte, se aprecia que A5 (salida de malos olores) ocurre 3 de cada 4 veces en el 'saneamiento colgado', que A1 (fugas en las conducciones) tiene una presencia de $2 / 3$ del total en este mismo subtipo de instalación, y finalmente, que el tipo de anomalía A3 (ausencia o deficiente disposición de piezas) se distribuye a partes iguales entre el 'saneamiento colgado' y el 'saneamiento enterrado'.

Tipos de anomalías por cada clase de saneamiento

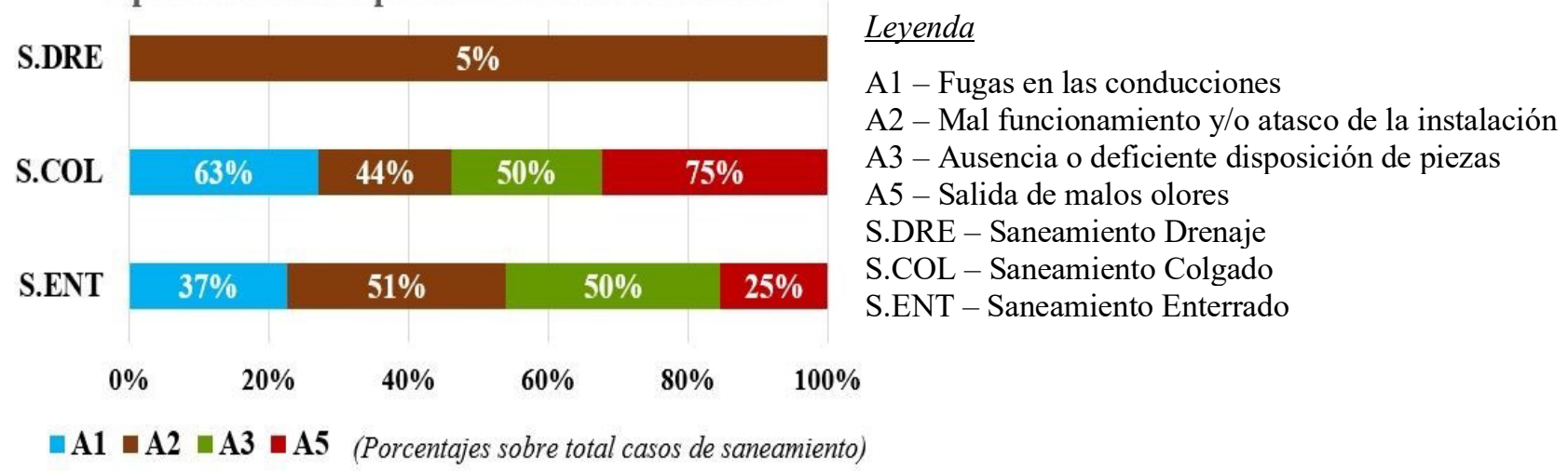

Figura 8. Distribución de casos por subtipo de la instalación de saneamiento y por tipo de anomalía. 


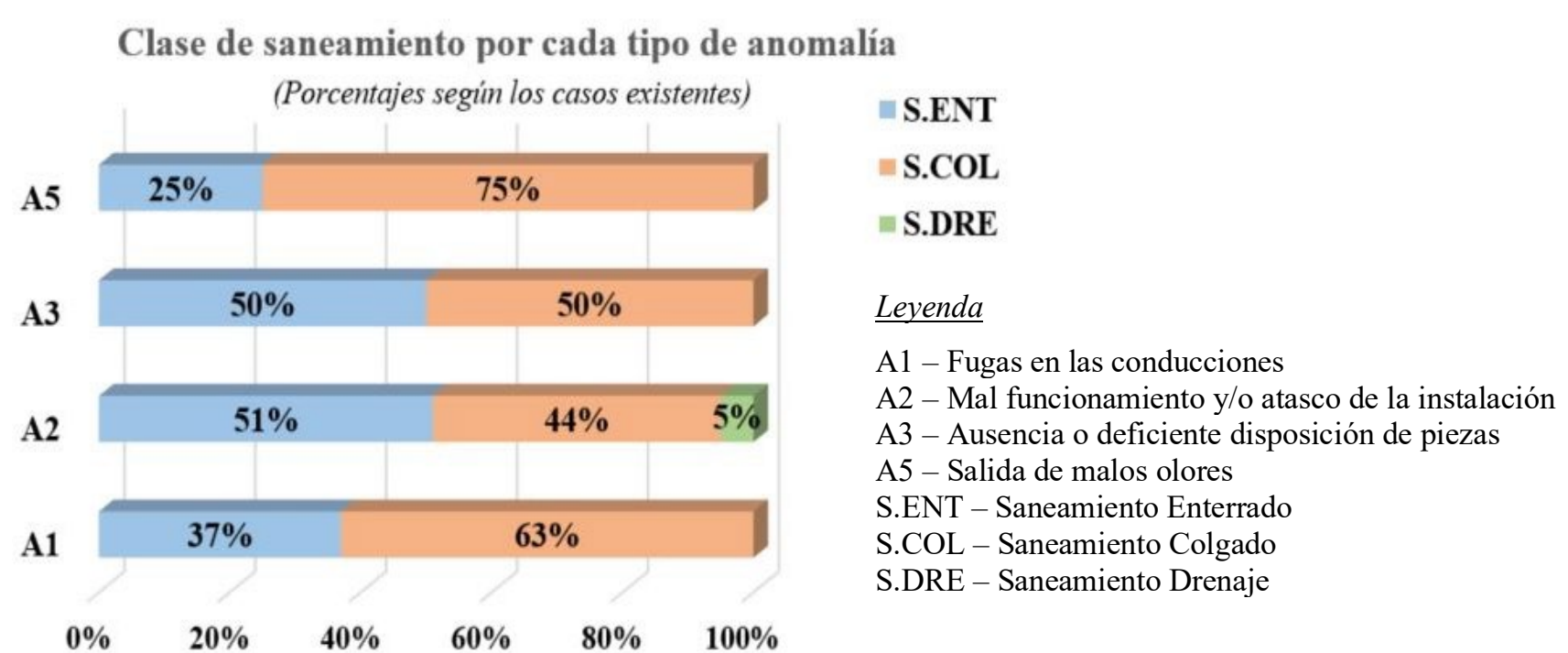

Figura 9. Distribución de casos por tipo de anomalía y por subtipo de la instalación de saneamiento.

\subsection{Número de casos orígenes del fallo por cada tipo de anomalía.}

Una de las informaciones más relevantes para los técnicos que tienen responsabilidades en la dirección de las obras, es conocer cada uno de los tipos de orígenes del fallo que dan lugar a cada uno de los tipos de anomalías. Esta información es extraordinariamente difícil encontrarla en las investigaciones, pues resulta complejo su constatación, y más aún, obtener un número de casos suficientes para que sea mínimamente representativo. En la Tabla 5 se proporcionan estas interrelaciones, lo que facilitará de manera importante la prevención de problemáticas en las obras, si se sabe implementar los procesos de mejoras en los sistemas constructivos para no repetir los mismos errores.

Tabla 5 - Relación de tipos de orígenes del fallo por cada uno de los tipos de anomalías

\begin{tabular}{|c|l|c|}
\hline Código & \multicolumn{1}{|c|}{ Concepto (Anomalía/Origen del fallo) } & No $^{\mathbf{c}}$ casos \\
\hline A1 & Fugas en las conducciones & $\mathbf{7 2}$ \\
\hline OF2 & Uniones/acoples deteriorados o mal colocados & 30 \\
\hline OF1 & Daño en la instalación & 22 \\
\hline OF3 & Falta de colocación y omisión de elementos & 8 \\
\hline OF5 & Inadecuado material o solución constructiva & 7 \\
\hline OF4 & Insuficiente porcentaje de pendiente & 4 \\
\hline OF6 & Mantenimiento disconforme & 1 \\
\hline A2 & Mal funcionamiento y/o atasco de la instalación & 47 \\
\hline OF1 & Daño en la instalación & 22 \\
\hline OF4 & Insuficiente porcentaje de pendiente & 9 \\
\hline OF2 & Uniones/acoples deteriorados o mal colocados & 4 \\
\hline OF5 & Inadecuado material o solución constructiva & 3 \\
\hline OF6 & Mantenimiento disconforme & 2 \\
\hline OF3 & Falta de colocación y omisión de elementos \\
\hline
\end{tabular}




\begin{tabular}{|c|l|c|}
\hline A3 & Ausencia o deficiente disposición de piezas & $\mathbf{2 3}$ \\
\hline OF3 & Falta de colocación y omisión de elementos & 19 \\
\hline OF1 & Daño en la instalación & 3 \\
\hline OF2 & Uniones/acoples deteriorados o mal colocados & 1 \\
\hline A4 & Desajustes y disfunciones & $\mathbf{1 0}$ \\
\hline OF1 & Daño en la instalación & 7 \\
\hline OF3 & Falta de colocación y omisión de elementos & 3 \\
\hline A5 & Salida de malos olores & $\mathbf{8}$ \\
\hline OF1 & Daño en la instalación & 4 \\
\hline OF3 & Falta de colocación y omisión de elementos & 3 \\
\hline OF2 & Uniones/acoples deteriorados o mal colocados & 1 \\
\hline
\end{tabular}

En base a dicha tabla, se puede documentar que: la anomalía A1 ocurre básicamente por la existencia de OF2, y en segundo lugar de OF1; que A2 se da mayoritariamente a causa de OF1; que A3 se da casi exclusivamente por OF3; que A4 está motiva principalmente por OF1; y que A5 no tiene un origen de fallo predominante.

\section{CONCLUSIONES}

Los informes técnicos periciales que se han tomado como base de datos para analizar las demandas realizadas por los usuarios, han demostrado ser un procedimiento de gran interés para determinar los tipos de anomalías y los orígenes del fallo de las instalaciones con canalizaciones de agua. De entre las cuatro instalaciones analizadas, el 'saneamiento' es la que más concentración de anomalías tiene, con un $72 \%$ del total.

De las tres tipologías constructivas que se estudiaron, las 'edificaciones en altura', son con diferencia en la que concurre un mayor número de casos $(62,74 \%)$, seguido de las viviendas 'unifamiliares pareadas/adosadas' (21,44\%).

De entre los tipos de anomalías determinadas, las 'fugas en las conducciones' es la más recurrente ( $\mathrm{A} 1=72$ de los 160 casos analizados en las instalaciones ICCA). Por su parte, el origen del fallo más frecuente es 'daño en la instalación' (OF1= 53 casos).

Debido a que las instalaciones de los edificios residenciales son cada vez más similares a nivel mundial, se considera que los resultados de este estudio pueden ser extrapolables a otros países del entorno europeo. Futuros trabajos de investigación pueden centrarse en cuantificar el valor económico de las anomalías de este tipo de instalaciones y evaluar mediante el análisis de ciclo de vida su influencia en el impacto ambiental del edificio.

\section{AGRADECIMIENTOS}

El presente trabajo se ha desarrollado dentro del Plan marco de actuación aprobado por la Fundación-Musaat, donde se acordó la realización de una investigación de ámbito nacional español sobre patologías en la edificación (Carretero-Ayuso and Moreno-Cansado, 2019). 


\section{GLOSARIO DE ABREVIACIONES}

\begin{tabular}{|c|c|}
\hline Abreviatura & Concepto o significado \\
\hline A1 & Fugas en las conducciones \\
\hline A2 & Mal funcionamiento y/o atasco de la instalación \\
\hline A3 & Ausencia o deficiente disposición de piezas \\
\hline A4 & Desajustes y disfunciones \\
\hline A5 & Salida de malos olores \\
\hline I1 & Saneamiento \\
\hline $\mathrm{I} 2$ & Fontanería \\
\hline $\mathrm{I} 3$ & Calefacción \\
\hline I4 & Protección contra incendios \\
\hline ICCA & Instalaciones Con Canalizaciones de Agua \\
\hline ISCA & Instalaciones Sin Canalizaciones de Agua \\
\hline OF1 & Daño en la instalación \\
\hline OF2 & Uniones/acoples deteriorados o mal colocados \\
\hline OF3 & Falta de colocación y/o omisión de elementos \\
\hline OF4 & Insuficiente porcentaje de pendiente \\
\hline OF5 & Inadecuado material o solución constructiva \\
\hline OF6 & Mantenimiento disconforme \\
\hline TO-1 & Edificación en altura \\
\hline TO-2 & Unifamiliar pareada/adosada \\
\hline TO-3 & Unifamiliar aislada \\
\hline S.COL & Saneamiento Colgado \\
\hline S.DRE & Saneamiento Drenaje \\
\hline S.ENT & Saneamiento Enterrado \\
\hline
\end{tabular}

\section{REFERENCIAS}

AENOR. (2012). " Norma UNE-EN 15331:2012. Criterios para el diseño, la gestión y el control de servicios de mantenimiento de edificios".

Arencibia, J. M. (2007). "Fundamental Concepts for the Maintenance of Buildings". Revista de Arquitectura e Ingeniería, 1, 1-8.

Azorín López, V., and Monjo Carrió, J. (2005). "La investigación en construcción. Conclusiones de las I Jornadas de Investigación en Construcción". Informes de la Construcción, 5(498), 4-15.

Blanchére, G. (1978). “Saber construir”. Apr-2003 Ed., Editores Técnicos Asociados, Sevilla.

Carretero-Ayuso, M. J., and Moreno-Cansado, A. (2019). Estudio Estadístico Nacional sobre Patologías en la Edificación, Fundación-Musaat, Madrid.

Chong, W., and Low, S. (2006). "Latent Building Defects: Causes and Design Strategies to Prevent Them". J. Perform. Constr. Facil., 20(3), 213-221.

Chou, J., Yang, I., Chong, W. K. (2009). "Probabilistic Simulation for Developing Likelihood Distribution of Engineering Project Cost." Automation in Construction, 18, 570-577. 
Fernández, J. L. (2015). "Un servicio pionero de los aparejadores ahorra miles de euros en los litigios por la vivienda". <http://www.abc.es/local-alicante/20150914/abci-litigios-viviendaservicio-201509141011.html?utm_content>.

Forcada, N., Macarulla, M., Love, P. E. D. (2013). "Assessment of Residential Defects at PostHandover". J. Constr. Eng. Manage., 139(4), 372-378.

Gallego Brizuela, C. (2011). La indemnización por defectos constructivos en el edificio y en la vivienda, CGB \& Asociados, Valladolid (España).

Garcez, N., Lopes, N., de Brito, J., Sá, G. (2012). "Pathology, Diagnosis and Repair of Pitched Roofs with Ceramic Tiles: Statistical Characterisation and Lessons Learned from Inspections". Constr. Build. Mater., 36(0), 807-819.

García Sanz-Calcedo, J. (2014). Mantenimiento eficiente de edificios, Agencia Extremeña de la Energía, Merida.

Josephson, P. E., and Hammarlund, Y. (1999). "The Causes and Costs of Defects in Construction: A Study of Seven Building Projects". Automation in Construction, 8(6), 681-687.

Lee, D., Chi, H., Wang, J., Wang, X., Park, C. (2016). "A Linked Data System Framework for Sharing Construction Defect Information using Ontologies and BIM Environments". Automation in Construction, 68, 102-113.

Martín-Gómez, C., Emmanuel, A., Jimenez, L., Murillo, M., Sagüés, R. (2006). "How Much Space is Occupied by the Installations of a Building?" El Instalador, 434, 5-49.

Meiss, A. E., and Muñoz, J. E. F. (2015). " Lesiones y rehabilitación de las instalaciones de fontanería y saneamiento en edificios destinados a viviendas". RE-Revista de Edificación, 45, 143151.

Min, Z., Morgenstern, P., Marjanovic-Halburd, L. (2016). "Facilities Management Added Value in Closing the Energy Performance Gap". International Journal of Sustainable Built Environment,

Ministerio de Fomento. (2015). Se constituye la comisión para la implantación de la metodología BIM, Ministerio of Fomento del Gobierno de España, Madrid.

MUSAAT. (2018). Expedientes e Informes Periciales de Siniestros de Construcción en España. Mutua de Aparejadores y Arquitectos Técnicos, Madrid.

OCU. (2018). "Complaints made in 2017." <http://www.ocu.org/organizacion/prensa/notas-deprensa/2017/reclamaciones-ante-la-ocu-en-2008435944> (feb/06, 2021).

Pauwels, P. (2014). "Supporting Decision-Making in the Building Life-Cycle using Linked Building Data". Buildings, 3, 549-579.

Sarman, A. M., Nawi, M. N., Che-Ani, A. I., Mazlan, E. M. (2015). "Concrete Flat Roof Defects in Equatorial Climates". International Journal of Applied Engineering Research, 10(3), 7319-7324.

SERJUTECA. (2013-2017). Expedientes y Documentación de Siniestros de Responsabilidad Civil Profesional de Aparejadores y Arquitectos Técnicos, Servicios Jurídicos Técnicos Aseguradores, Madrid.

WWF-Spain. (2010). Potential for Energy Savings and Reduction in CO2 Emissions of the Building Stock in Spain in 2020, WWF/Adena Ed., WWF/Adena, Madrid. 